\title{
Use of an over-the-scope clip for endoscopic sealing of a gastric fistula after sleeve gastrectomy
}

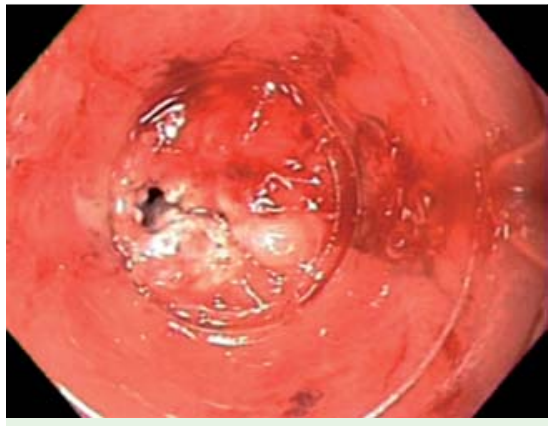

Fig. 1 Endoscopic fistula.

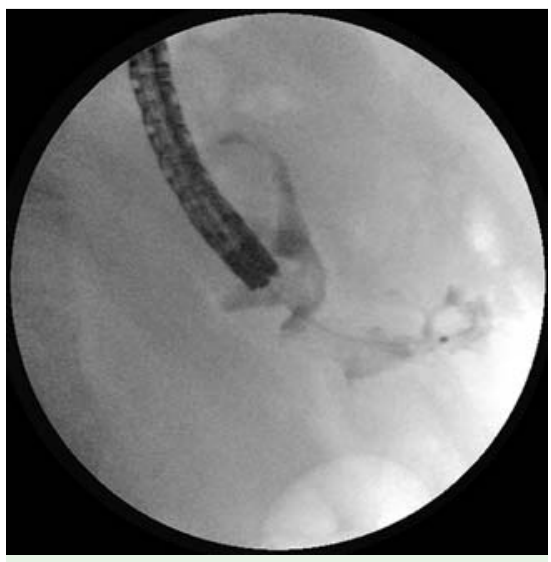

Fig. 2 X-ray examination showing the extravasion of contrast medium.

Perforation or development of a fistula in the gastrointestinal tract is a serious complication. A gastrocutaneous fistula after sleeve gastrectomy is difficult to treat, with a mortality rate of $85 \%$ following unsuccessful treatment [1]. These fistulas have been successfully managed with endoscopic fibrin sealing [2]. Preliminary experience with the over-the-scope clipping system (Ovesco, Tubingen, Germany) has shown the efficacy of this intervention in the management of severe bleeding and perforations of the gastrointestinal tract [3-5].

This is the first report of the use of an over-the-scope clipping for the management of a gastric fistula. A 43-year-old woman underwent sleeve gastrectomy for morbid obesity. After 1 week, a fistula developed at the proximal end of the suture, $2 \mathrm{~cm}$ distal to the esophagogastric junction. A nasogastric drain and an ultrasound-guided external drain were insert-

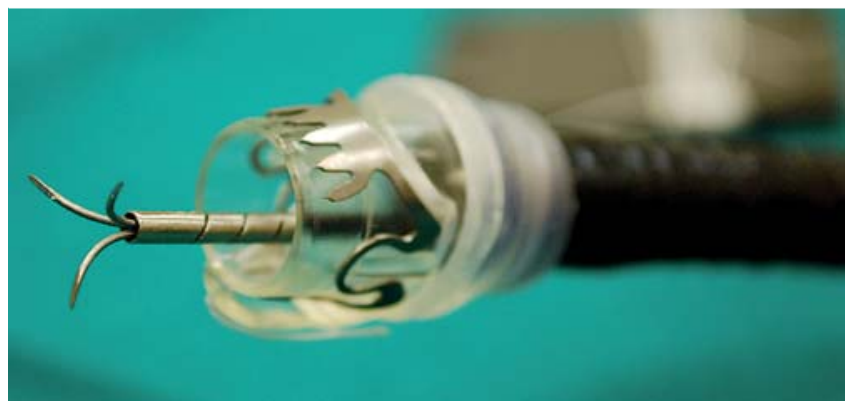

Fig. 3 The over-thescope clipping system with the anchor catheter.

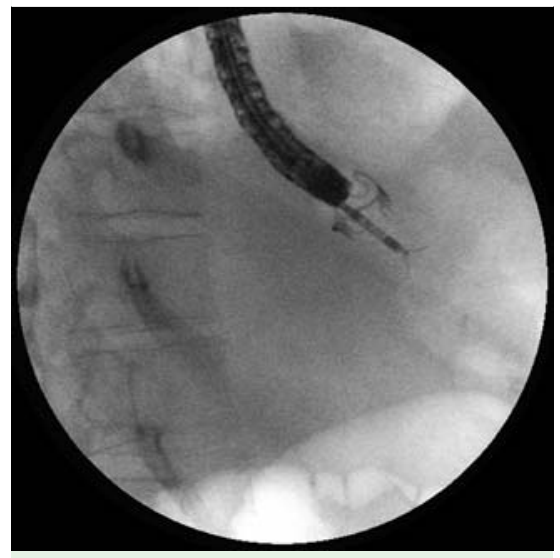

Fig. 4 The anchor catheter introduced through the fistula.

ed. Endoscopy showed a 7-mm orifice ( Fig. 1). Fig. 2 shows the extravasion of contrast medium, confirming the presence of a fistula. Two attempts to seal the fistula with hemoclips failed.

Placement of a large, colorectal expandable covered stent (Taewoong, Korea) was also attempted. The patient could not eat and complained of severe epigastric pain. The stent was removed 3 weeks later. Surgery was carried out, but the fistula reappeared 1 week later and a gastrocutaneous fistula was diagnosed.

The over-the-scope clipping system was used to overcome the limitations presented by the available hemoclips. The overthe-scope clip is delivered by means of an applicator cap placed on the tip of the endoscope. A catheter with a retractable anchor was introduced through the fistula and the grasped tissue firmly pulled inside the cap ( Fig. 3 and 4 ).

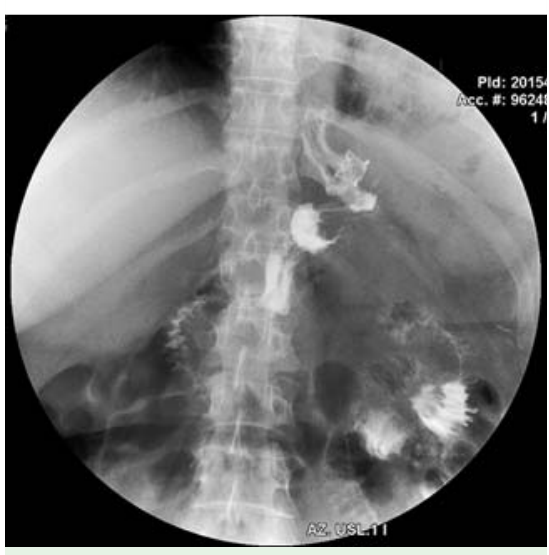

Fig. 5 Fluoroscopic control after treatment with the over-the-scope clipping system.

The clip was then released and the fistula successfully closed. The patient was allowed to eat her usual diet 24 hours later after post-treatment evaluation. She was then discharged. The endoscopic and radiologic controls performed after 1 and 2 weeks confirmed sealing of the fistula (๑ Fig. 5).

\section{Endoscopy_UCTN_Code_TTT_1AO_2AI}

\section{Conio ${ }^{1}$, S. Blanchi ${ }^{1}$, A. Repici²,}

R. Bastardini ${ }^{1}$, G. M. Marinari ${ }^{3}$

Department of Gastroenterology, General Hospital, Sanremo, Italy

2 Digestive Endoscopy Unit, IRCCS Istituto Clinico Humanitas, Milan, Italy 3 Department of Surgery, Unit of Obesity Surgery, AOU S. Martino Hospital, Genova, Italy 


\section{References}

1 Rolandelli R, Roslyn JJ. Surgical management and treatment of sepsis associated with gastrointestinal fistulas. Surg Clin North Am 1996; 76: $1111-1122$

2 Papavramidis TS, Kotzampassi K, Kotidis E et al. Endoscopic fibrin sealing of gastrocutaneous fistulas after sleeve gastrectomy and biliopancreatic diversion with duodenal switch. J Gastroenterol Hepatol 2008; 23: $1802-1805$

3 Kratt T, Küper $M$, Traub F et al. Feasibility study for secure closure of natural orifice transluminal endoscopic surgery gastrotomies by using over-the-scope clips. Gastrointest Endosc 2008; 68: 993 - 996

4 Kirschniak A, Kratt T, Stüker D et al. A new endoscopic over-the-scope clip system for treatment of lesions and bleeding in the GI tract: first clinical experiences. Gastrointest Endosc 2007; 66: $162-167$

5 Kirschniak A, Traub F, Kueper MA et al. Endoscopic treatment of gastric perforation caused by acute necrotizing pancreatitis using over-the-scope clips: a case report. Endoscopy 2007; 39: 1100-1102
Bibliography

DOI $10.1055 / \mathrm{s}-0029-1215199$

Endoscopy 2010; 42: E71 - E72

(C) Georg Thieme Verlag KG Stuttgart · New York · ISSN 0013-726X

\section{Corresponding author}

\section{Conio, MD}

Department of Gastroenterology

C.so Garibaldi 187, 3

Sanremo

Italy

Fax: +39-184-536875

mxconio@tin.it 